\section{OPEN ACCESS}

Edited by:

David Pozo,

Universidad de Sevilla, Spain

Reviewed by:

Ruoyu Zhang,

Zhejiang University, China

Cintia Roodveldt,

Centro Andaluz de Biología Molecular

y Medicina Regenerativa

(Cabimer), Spain

*Correspondence:

Francisco Capan

franciscocapani@hotmail.com

†These authors have contributed equally to this work

Specialty section:

This article was submitted to

Neurodegeneration,

a section of the journal

Frontiers in Neurology

Received: 01 May 2019

Accepted: 16 September 2019

Published: 09 October 2019

Citation:

Herrera MI, Kölliker-Frers RA,

Otero-Losada M, Perez Lloret S,

Filippo M, Tau J, Capani F and

Villa AM (2019) A Pilot Cross-Sectional

Study to Investigate the Biomarker

Potential of Phosphorylated

Neurofilament-H and Immune

Mediators of Disability in Patients With

5 Year Relapsing-Remitting Multiple

Sclerosis. Front. Neurol. 10:1046.

doi: 10.3389/fneur.2019.01046

\title{
A Pilot Cross-Sectional Study to Investigate the Biomarker Potential of Phosphorylated Neurofilament-H and Immune Mediators of Disability in Patients With 5 Year Relapsing-Remitting Multiple Sclerosis
}

\begin{abstract}
María Inés Herrera ${ }^{1,2 \dagger}$, Rodolfo Alberto Kölliker-Frers ${ }^{1,3 \dagger}$, Matilde Otero-Losada ${ }^{1}$, Santiago Perez Lloret ${ }^{1}$, Macarena Filippo ${ }^{2}$, Julia Tau ${ }^{4}$, Francisco Capani ${ }^{1,5,6,7 *}$ and Andrés M. Villa ${ }^{8}$

1 Instituto de Investigaciones Cardiológicas, National Research Council, Universidad de Buenos Aires (ININCA, UBA-CONICET), Buenos Aires, Argentina, ${ }^{2}$ Centro de Investigaciones en Psicología y Psicopedagogía, Pontificia Universidad Católica Argentina (CIPP, UCA), Buenos Aires, Argentina, ${ }^{3}$ Facultad de Farmacia y Bioquímica, Universidad Maimónides, Buenos Aires, Argentina, ${ }^{4}$ Laboratorio de Investigación Ocular, Departamento de Patología, Facultad de Medicina, Universidad de Buenos Aires, Buenos Aires, Argentina, ${ }^{5}$ Departamento de Biología, Universidad John F. Kennedy, Buenos Aires, Argentina, ${ }^{6}$ Facultad de Psicología, Pontificia Universidad Católica Argentina UCA, Buenos Aires, Argentina, ${ }^{7}$ Instituto de Ciencias Biomédicas, Facultad de Ciencias de la Salud, Universidad Autónoma de Chile, Santiago, Chile,

${ }^{8}$ Seccion de Neuroinmunología Clínica, Servicio de Neurología, Hospital José María Ramos íMejía, Buenos Aires, Argentina
\end{abstract}

Objective: To test the feasibility of conducting a full-scale project evaluating the potential value of the phosphorylated neurofilament $\mathrm{H}(\mathrm{pNF}-\mathrm{H})$ and several cytokines as disability markers in relapsing-remitting multiple sclerosis (RRMS).

Methods: Twenty-four patients with 5-year RRMS evolution and eleven healthy control subjects entered the study. None of the participants had an inflammatory systemic or metabolic disease. Disability progression was evaluated using the Expanded Disability Status Scale. Serum level of pNF-H, the anti-inflammatory cytokine transforming growth factor- $\beta 1$ (TGF- $\beta 1$ ), and the pro-inflammatory cytokines

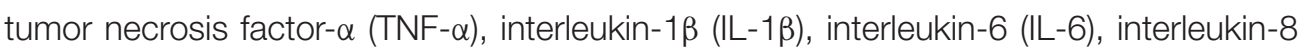
(IL-8), interleukin-17A (IL-17A), monocyte chemotactic protein-1 (MCP-1), and soluble intercellular cell-adhesion molecule 1 (SICAM-1) were quantified using enzyme-linked immunosorbent assay (ELISA).

Results: The patients had higher serum level of TGF- $\beta 1, \| L-6$, sICAM- 1 , and pNF-H. Based on these findings, a sample of at least 49 controls and 89 recent-onset RRMS patients is required to find an at least 1-point between-group difference in pNF-H with a power of $80 \%$ and an $\alpha$ error $=0.05$. The progression of the disease was correlated with the level of pNF-H (Spearman rho $=0.624, p=0.006)$, but not with the cytokines'. 


\begin{abstract}
Conclusions: The serum level of pNF-H, EDSS score-correlated, might stand for a potential biomarker of disability in RRMS reflecting progressive axonal damage and cumulative neurological deterioration. The novelty of these results warrants conducting a larger confirmatory trial.
\end{abstract}

Keywords: relapsing-remitting multiple sclerosis, biomarker potential, neurofilament heavy chain phosphoform, cytokines, disability progression, expanded disability status scale

\section{INTRODUCTION}

Multiple sclerosis is the most prevalent chronic autoimmune disease of the central nervous system with the first clinical symptoms typically appearing in early adulthood, and affecting over 2 million people worldwide (1). Relapsing-remitting multiple sclerosis (RRMS) is the current criteria-based most prevalent form of the three clinical presentations of multiple sclerosis (MS), relapsing-remitting, converted to secondary progressive, and primary progressive. It is characterized by neurologic worsening attacks or relapses followed by apparent recovery periods of masked progression of the disease (2).

The identification of a reliable biomarker of the progressive axonal degeneration is critical to prevent long-term disability in RRMS. Biomarker dynamics in blood and CSF are poorly characterized in RRMS (3), and serum levels of the neurofilament heavy chain phosphoform (pNF-H) might provide surrogate information about ongoing neurodegeneration in RRMS (4). Likewise, the differential expression of cytokines directly involved in RRMS pathogenesis might also indicate disease status. However, their biomarker potential (5) has been validated for application in clinical practice only in a few cases. We conducted a pilot cross-sectional study to investigate the biomarker potential of $\mathrm{pNF}-\mathrm{H}$ and several cytokines for disability in patients with 5-year RRMS evolution. The control group included age-matched healthy subjects. Provided the purported biomarkers reflected the patients' disability degree, their detection in blood might promise useful for early diagnosis, and monitoring of the therapeutic response.

Being RRMS an orphan disease makes recruiting large patient samples difficult, time-consuming, and expensive. Therefore, given the clinical relevance of this topic, we resorted to carrying out the present pilot, preliminary small-case study, to explore the feasibility of conducting a large-scale trial to compare the levels of the pNF-H and cytokines in patients with RRMS and healthy controls using the present protocol, based on the coming out information (6-8).

\section{METHODS}

\section{Study Design}

An observational cross-sectional, transverse analysis was conducted (2009-2012). The ethics committees of the Faculty of Medicine, University of Buenos Aires (UBA) and the José María Ramos Mejía Hospital (JMRMH) approved the protocol. All participants were informed and gave written consent.

\section{Subjects}

Twenty-four RRMS drug-naive patients with a 5-year disease evolution and 11, age-matched healthy controls (age range 3060 y. o.) completed a validated questionnaire on personal characteristics, medical history, smoking, diet, alcohol consumption, and current medication. Blood samples were obtained during the 3-month relapse period when the pNF-H concentration is expected to increase. The control group underwent neurological and laboratory examination at the Neurology Service of the JMRMH for inclusion purposes only.

\section{Inclusion Criteria for Patients}

- Patients over 18 years old with a 5-year RRMS evolution and early diagnosis of the disease according to the McDonald criteria.

- EDSS value $<5.5$.

\section{Inclusion Criteria for Controls}

- Healthy volunteers over 18 years old.

\section{Exclusion Criteria for Patients and Controls}

- History of major psychiatric disorders.

- Pregnancy or lactation.

- Any systemic disease: current infection or chronic disease, autoimmune diseases except for RRMS in the patients, atherosclerosis, diabetes, metabolic syndrome, etc.

\section{Sampling}

Blood samples were collected from patients (during remissions) and controls after $12 \mathrm{~h}$ overnight fasting. Blood samples were left at room temperature for $30 \mathrm{~min}$ until clot formation, and sera were separated and stored at $-20^{\circ} \mathrm{C}$ until determinations. All participants underwent magnetic resonance imaging (MRI) recording.

\section{Neurofilament Heavy Chain Phosphoform (pNF-H) Determination}

A specific sandwich ELISA kit (NS170, Chemicon International, USA-CANADA) was used to quantify pNF-H. A special ELISA 96-well chicken anti- pNF-H polyclonal antibody precoated immunoplate was used to capture pNF-H which was detected using rabbit anti- pNF-H polyclonal antibody $(1: 100)$, followed by an alkaline phosphatase-conjugated (1:2000) goat anti-rabbit polyclonal antibody. After pNPP (p-nitrophenyl phosphatase) alkaline phosphatase substrate addition, pNF-H was quantified by absorbance at $405 \mathrm{~nm}$. Limit of detection (LOD) $0.0585 \mathrm{ng} / \mathrm{mL}$, range of quantitation (ROQ): 0.0293 $\mathrm{ng} / \mathrm{mL}-15 \mathrm{ng} / \mathrm{mL}$. 


\section{Pro-inflammatory and Anti-inflammatory Molecules Determination}

To rule out traditional cardiovascular risk markers (CVRM), ELISA kits allowed quantifying the cytokines TNF- $\alpha$, IL-1 $\beta$, IL-6, IL-8, IL-17A, and TGF- $\beta 1$ (respectively 555212, 557953, 555220, 555244, Platinum, and 559119 BD Biosciences, New Jersey, USA), and sICAM-1 and MCP-1 (respectively Human Quantikine sICAM-1/CD54 and Human Quantikine MCP-1 R\&D Systems, Minneapolis, USA). LOD: $7.8 \mathrm{pg} / \mathrm{mL}$ for TNF$\alpha, 3.9 \mathrm{pg} / \mathrm{mL}$ for IL- $1 \beta, 4.7 \mathrm{pg} / \mathrm{mL}$ for IL-6, $3.1 \mathrm{pg} / \mathrm{mL}$ for IL-8, $1.6 \mathrm{pg} / \mathrm{mL}$ for IL-17A, $4.7 \mathrm{pg} / \mathrm{mL}$ for TGF- $\beta 1,1.56 \mathrm{ng} / \mathrm{mL}$ for sICAM-1, and $2.31 \mathrm{pg} / \mathrm{mL}$ for MCP-1.

In all cases, ELISA kits were used according to technical instructions and following technical specifications.

\section{TNF- $\alpha$ Assay Kit}

The Human TNF- $\alpha$ (555212), ELISA kit (BD Biosciences, New Jersey, USA) is an in-vitro ELISA for the quantitative measurement of human TNF- $\alpha$ in sera. The lower limit of detection is $7.8 \mathrm{pg} / \mathrm{mL}$ (the lowest positive standard value).

\section{IL-1 $\beta$ Assay Kit}

The Human IL-1 $\beta$ (557953), ELISA kit (BD Biosciences, New Jersey, USA) is an in vitro ELISA for the quantitative measurement of human IL-1 $\beta$ in sera. The lower limit of detection is $3.9 \mathrm{pg} / \mathrm{mL}$ (the lowest positive standard value).

\section{IL-6 Assay Kit}

The Human IL-6 (555220), ELISA kit (BD Biosciences, New Jersey, USA) is an in-vitro ELISA for the quantitative measurement of human IL-6 in sera. The lower limit of detection is $4.7 \mathrm{pg} / \mathrm{mL}$ (the lowest positive standard value).

\section{IL-8 Assay Kit}

The Human IL8 (555244) ELISA kit (BD Biosciences, New Jersey, USA) is an in-vitro ELISA for the quantitative measurement of human IL-8 in sera. The lower limit of detection is $3.1 \mathrm{pg} / \mathrm{mL}$ (the lowest positive standard value).

\section{IL-17A6 Assay Kit}

The Human IL-17A Platinum ELISA kit (BD Biosciences, New Jersey, USA) is an in vitro ELISA for the quantitative measurement of human IL-17A. The lower limit of detection is $1.6 \mathrm{pg} / \mathrm{mL}$ (the lowest positive standard value).

\section{TGF- $\beta 1$ Assay Kit}

The Human TGF- $\beta 1$ (559119), ELISA kit (BD Biosciences, New Jersey, USA) is an in vitro ELISA for the quantitative measurement of human TGF- $\beta 1$ in sera. The lower limit of detection is $4.7 \mathrm{pg} / \mathrm{mL}$ (the lowest positive standard value).

\section{sICAM-1 Assay Kit}

The Human Quantikine sICAM-1/CD54 ELISA kit (R\&D Systems, Minneapolis, United States of America) is an in-vitro ELISA for the quantitative measurement of human sICAM-1 in sera, plasma and cell culture supernatants. Sera were used to determine sICAM-1 levels according to technical specifications.
The lower limit of detection is $1.56 \mathrm{ng} / \mathrm{mL}$ (the lowest positive standard value).

\section{MCP-1 Assay Kit}

The Human Quantikine MCP-1 ELISA kit (R\&D Systems, Minneapolis, United States of America) is an in-vitro ELISA for the quantitative measurement of human MCP-1 in sera, plasma and cell culture supernatants. Sera were used to determine MCP1 levels according to technical specifications. The lower limit of detection is $2.31 \mathrm{pg} / \mathrm{mL}$ (the lowest positive standard value).

\section{Disability Assessment Using the Expanded Disability Status Scale (EDSS)}

The worldwide used Expanded Disability Status Scale (EDSS) (9) for assessing neurological impairment and disability progression evaluates:

- Pyramidal weakness or difficulty in moving limbs;

- Cerebellar ataxia;

- Loss of coordination or tremor;

- Brainstem problems with speech, swallowing, and nystagmus;

- Sensory numbness or loss of sensations;

- Bowel and bladder dysfunction;

- Visual dysfunction;

- Mental dysfunction.

Scale grading: $0=$ normal neurological exam, $1.0-4.5=$ ambulatory patients, $5.0-9.5=$ impaired ambulation, and $10=$ death by RRMS (8).

\section{Statistical Analysis}

Non-parametric tests were used due to the non-Gaussian data distribution and the small sample size. The Mann-Whitney $U$ test yielded between-groups comparison, and the Spearman's Rho method evaluated correlations. Assessment of possible differences between patients native or referred to our tertiary center was performed by logistic regression. The alpha error was set at 0.05 (SPSS 23.0, NY, USA). The reduced number of cases studied precluded us from conducting a multivariate analysis to discard potential confounding factors. All the same, controls and patients were fairly indistinguishable as for gender and age.

TABLE 1 | Neuroimmune markers in RRMS patients and healthy controls.

\begin{tabular}{lccr}
\hline & Controls $(\boldsymbol{n}=\mathbf{1 1})$ & RRMS patients $(\boldsymbol{n}=\mathbf{2 4})$ & $\boldsymbol{p}$-value \\
\hline PNF-H & $0.16(0.15 ; 0.18)$ & $3.17(2.13 ; 5.37)$ & $<0.001$ \\
IL-17A & $5.44(4.22 ; 8.20)$ & $8.28(6.77 ; 9.72)$ & 0.055 \\
TGF- $\beta 1$ & $80.00(39.00 ; 150.00)$ & $1087.00(557.00 ; 2,417.00)$ & $<0.001$ \\
IL-1 $\beta$ & $4.30(2.70 ; 10.53)$ & $7.38(3.64 ; 15.77)$ & 0.296 \\
TNF- $\alpha$ & $3.00(1.56 ; 6.00)$ & $4.33(1.67 ; 8.78)$ & 0.497 \\
IL-6 & $7.47(3.87 ; 9.00)$ & $21.00(14.93 ; 35.46)$ & $<0.001$ \\
IL-8 & $8.89(4.12 ; 13.70)$ & $18.63(11.70 ; 41.00)$ & $<0.019$ \\
MCP-1 & $131.00(108.00 ; 157.63)$ & $162.00(112.63 ; 211.00)$ & 0.207 \\
sICAM-1 & $222.04(212.78 ; 252.90)$ & $288.70(246.11 ; 444.00)$ & 0.015
\end{tabular}

Values are shown as median (25th; 75 th percentiles). 


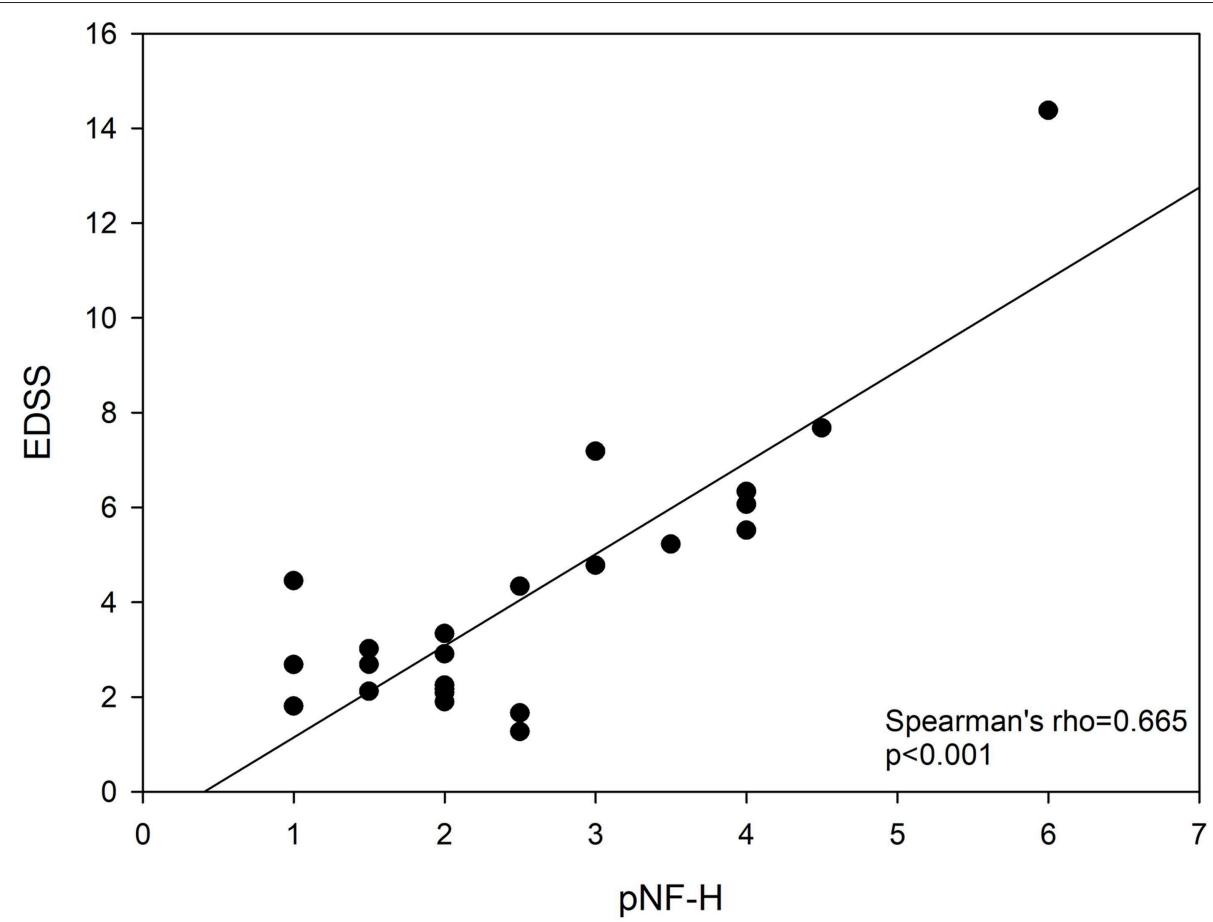

FIGURE 1 | Relationship between EDSS and pNF-H. EDSS, Expanded Disability Status Scale; pNF-H, phosphorylated neurofilament H.

\section{RESULTS}

Eleven healthy controls and 24 RRMS patients were recruited (43 and $39 \%$ of males respectively, $p=0.645$ ). Median (25th; 75 th percentiles) age was 38 years old $(33 ; 53)$ for controls and 39 years old $(31 ; 43)$ for patients $(p=0.699)$. The patients' group had a median EDSS score of $2.3(2.0 ; 3.5)$, minimum and maximum scores were 1 and 6 . Five patients were referred from other centers.

The RRMS patients had higher plasmatic levels of pNF-H, TGF- $\beta 1$, IL-6, and sICAM-1 (Table 1). In RRMS patients, EDSS score was not related with IL-17A (rho $=0.094, p=0.760, n$ $=13$ ), TGF- $\beta 1$ (rho $=0.052, p=0.880, n=11)$, IL-1 $\beta$ (rho $=-0.114, p=0.711, n=13)$, TNF- $\alpha$ (rho $=-0.168, p=$ $0.583, n=13$ ), IL-6 (rho $=0.125, p=0.683, n=13$ ), IL-8 (rho $=-0.316, p=0.293, n=13$ ), MCP-1 (rho $=0.274, p=$ $0.476, n=9$ ), and sICAM-1 (rho $=0.170, p=0.688, n=8$ ). Conversely, pNF-H correlated with EDSS score (rho $=0.624$, $p=0.006, n=18$, Figure 1). A logistic regression analysis ruled out any confounding effect due to the patients' referring center $(p=0.705)$.

\section{DISCUSSION}

We have observed a median 3-point difference in pNF-H value between RRMS patients and healthy controls. Heterogeneity regarding disease severity due to the contribution of mild to intermediate RRMS patients in this study might account for data dispersion and contribute to concealing between-group differences. To better understand the value of determining serum
pNF-H level, a clinical study should include at least 49 controls and 89 recent-onset RRMS patients to find an at least 1-point between-group difference in pNF with a power of $80 \%$ and an $\alpha$ error $=0.05$, based on the variability parameters calculated in this study. Our study showed a correlation between the pNF$\mathrm{H}$ level and disease progression, but multivariate analyses to identify possible confounding factors were not possible due to the reduced number of cases. Typically, these studies require 200-300 patients with varying disease evolution.

Unlike inflammatory cytokines, the serum level of pNF-H (axonal damage) correlated with EDSS score values, suggesting its likely reliability in monitoring the clinical condition in RRMS patients with 0-5.5 EDSS score values.

A study by Trapp and colleagues showed that axonal pNF$\mathrm{H}$ staining was more frequent in MS patients than in healthy controls (10). Thereafter, pNF-H has been proposed as a surrogate marker of axonal injury in many neurodegenerative diseases (11). In MS, while cerebrospinal fluid or blood phosphorylated light chain neurofilament level has been proposed as a useful clinical biomarker $(12,13)$, results on pNF$\mathrm{H}$ are scarce. Patients with a high pNF-H level are exposed to an evolutionary disability risk higher than those with high TGF$\beta 1$, IL-6, and sICAM-1, which showed a poorer relationship with disability.

Cytokines reflect an inflammatory state, which makes up part of a complex process of neuronal death (14). The lack of correlation between cytokine levels and EDSSscore reinforces the idea that no single cytokine has so far emerged as an undisputed biomarker candidate of disability state though they should be confirmed in a 
larger number of patients. Regardless of the unquestionable immunopathogenic role of cytokines in RRMS, their fluctuations add complexity to the inherent uncertainty derived from inflammatory outbreaks and remissions over time. Idiosyncrasy concerning inflammation characteristics and the individual response to treatment precludes cytokines from embodying a good marker of evolutionary risk. The increase in TGF $\beta-1$ level in RRMS patients agrees with the clinical remission phase they were at the time of blood sampling.

Petzold (15) reported an increase in pNF-H level in patients with progressive disease. The positive correlation found between serum pNF-H and the EDSS score in patients with a 5-year RRMS evolution in this study may well serve as the cornerstone on the way to validation.

The outcome of this pilot study provides empirical evidence to put forward pNF-H and certain cytokines as likely RRMS biomarkers. Keeping in mind Carl Sagan' statement on absence of evidence is not evidence of absence, noteworthy the serum level of pNF-H (axonal damage) unlike serum cytokines (inflammation) correlated with EDSS score. Our data show that determination of pNF-H and cytokines complement each other in RRMS. Currently, we pursue developing a neuroinflammation score increasing their conjoint predictive power.

\section{CONCLUSION}

A large clinical study to better understand the value of the pNF as a prognostic, early marker of MS disability progression is feasible using the currently used protocol. Likewise, it may aid the neurologist in customizing the treatment. Based on the variability parameters observed in this study, it should include at least 49 controls and 89 recent-onset RRMS patients to find an at least 1point between-group difference in pNF with a power of $80 \%$ and an $\alpha$ error $=0.05$. A suitable full-scale multicenter study should

\section{REFERENCES}

1. Reich DS, Lucchinetti CF, Calabresi PA. Multiple clerosis. $N$ Engl J Med. (2018) 378:169-80. doi: 10.1056/NEJMra14 01483

2. Weiner HL. The challenge of multiple sclerosis: how do we cure a chronic heterogeneous disease? Ann Neurol. (2009) 65:239-48. doi: 10.1002/ana. 21640

3. Katz R. Biomarkers and surrogate markers: an FDA perspective. NeuroRx. (2004) 1:189-95. doi: 10.1602/neurorx.1.2.189

4. Büki A, Povlishock JT. All roads lead to disconnection? Traumatic axonal injury revisited. Acta Neurochir. (2006) 148:181-94. doi: 10.1007/s00701-005-0674-4

5. Alves-Leon SV, Batista E, Papais-Alvarenga R, Quírico-Santos T. Determination of soluble ICAM-1 and TNF- $\alpha$ R in the cerebrospinal fluid and serum levels in a population of Brazilian patients with relapsing-remitting multiple sclerosis. Arq Neuropsiquiatr. (2001) 59:18-22. doi: 10.1590/S0004-282X20010001 00005

6. Julious SA. Sample size of 12 per group rule of thumb for a pilot study. Pharm Stat. (2005) 4:287-91. doi: 10.1002/pst.185 conveniently evaluate both early diagnosed and advanced-stage RRMS patients, with a thorough evaluation of the disease, and follow-up if possible.

\section{LIMITATIONS OF THIS STUDY}

This pilot study evaluated a small sample of patients. This resulted in a lack of statistical power, inability to conduct multivariate analyses, and poor generalizability beyond the reduced number of cases studied.

\section{DATA AVAILABILITY STATEMENT}

The datasets generated for this study are available on request to the corresponding author.

\section{ETHICS STATEMENT}

The ethics committees of the Faculty of Medicine, University of Buenos Aires and the José María Ramos Mejía Hospital approved the protocol. All participants were informed and gave written consent.

\section{AUTHOR CONTRIBUTIONS}

MH: writing. RK-F: original idea and investigation. MO-L: review and editing - grammar, style, and language. SP: statistical analysis, table, and figures. MF and JT: investigation. FC: funding acquisition and supervision. AV: investigation and supervision.

\section{FUNDING}

This work was supported by a PIDC 0031 2014-2020 grant from the National Agency for Scientific and Technological Promotion (ANPCyT), Argentina.
7. Lancaster GA, Dodd S, Williamson PR. Design and analysis of pilot studies: recommendations for good practice. J Eval Clin Pract. (2004) 10:307-12. doi: 10.1111/j..2002.384.doc.x

8. Moore CG, Carter RE, Nietert PJ, Stewart PW. Recommendations for planning pilot studies in clinical and translational research. Clin Transl Sci. (2011) 4:332-7. doi: 10.1111/j.1752-8062.2011. 00347.x

9. Kurtzke JF. Rating neurologic impairment in multiple sclerosis: an expanded disability status scale (EDSS). Neurology. (1983) 33:1444-52. doi: 10.1212/WNL.33.11.1444

10. Trapp BD, Peterson JP, Ransohoff RM, Rudick R, Mörk S, Bö L. Axonal transection in the lesions of multiple sclerosis. N Engl J Med. (1998) 338:27885. doi: 10.1056/NEJM199801293380502

11. Polman $\mathrm{CH}$, Reingold SC, Banwell B, Clanet M, Cohen JA, Filippi M, et al. Diagnostic criteria for multiple sclerosis: 2010 revisions to the McDonald criteria. Ann Neurol. (2011) 69:292-302. doi: 10.1002/ana. 22366

12. Sormani MP, Haering DA, Kropshofer H, Leppert D, Kundu U, Barro C, et al. Blood neurofilament light as a potential endpoint in Phase 2 studies in MS. Ann Clin Transl Neurol. (2019) 6:1081-9. doi: 10.1002/ac n3.795 
13. Martin SJ, McGlasson S, Hunt D, Overell J. Cerebrospinal fluid neurofilament light chain in multiple sclerosis and its subtypes: a meta-analysis ofcase-control studies. J Neurol Neurosurg Psychiatry. (2019) 90:1059-67. doi: 10.1136/jnnp-2018-319190

14. Ransohoff RM, Schafer D, Vincent A, Blachère NE, Bar-Or A. Neuroinflammation: ways in which the immune system affects the brain. Neurotherapeutics. (2015) 12:896-909. doi: 10.1007/s13311-01 5-0385-3

15. Petzold A. Neurofilament phosphoforms: surrogate markers for axonal injury, degeneration and loss. J Neurol Sci. (2005) 233:183-98. doi: 10.1016/j.jns.2005.03.015
Conflict of Interest: The authors declare that the research was conducted in the absence of any commercial or financial relationships that could be construed as a potential conflict of interest.

Copyright (c) 2019 Herrera, Kölliker-Frers, Otero-Losada, Perez Lloret, Filippo, Tau, Capani and Villa. This is an open-access article distributed under the terms of the Creative Commons Attribution License (CC BY). The use, distribution or reproduction in other forums is permitted, provided the original author(s) and the copyright owner(s) are credited and that the original publication in this journal is cited, in accordance with accepted academic practice. No use, distribution or reproduction is permitted which does not comply with these terms. 